\title{
In response to the article "Main pulmonary artery-to-descending aorta ratio in computed tomography: cut-off value to diagnose pulmonary hypertension in children". Pol J Radiol 2021; 86: e87-e92
}

\author{
Surya Pratap Singh ${ }^{\mathrm{E}}$, Sreenivasa Narayana Raju, Amarinder Singh ${ }^{\mathrm{E}}$ \\ All India Institute of Medical Sciences, New Delhi, Delhi, India
}

\section{Dear Editor,}

The article by Saetung et al. [1] on 'main pulmonary artery-to-descending aorta ratio in computed tomography' was quite informative and made for interesting reading.

The pulmonary artery to aorta ratio (PA/A) has been considered a proxy for pulmonary hypertension (PHT) and has been validated in many previous studies [2-4] not only for diagnosis but also for prognostication of patients [4].

The ascending aorta has been used most commonly for calculation of the pulmonary artery to aorta ratio and is well-validated; however, in cases where ascending aortic diameters cannot be used, an alternate aortic reference diameter is needed. The authors have identified the de- scending thoracic aortic diameter as an alternate aortic reference diameter with high specificity (91\%) and PPV (88\%).

Proximal aortic pathologies (arch hypoplasia, aortic interruption, coarctation of aorta) and involvement of DTA (e.g. vasculitis) can effect DTA diameters precluding use of DTA diameter. However, DTA diameters should only be used in cases where ascending aortic diameters cannot be used due to pathological involvement, and they can be a valuable alternative in this case. Considering the lack studies validating the MPA to DTA ratio and the small sample size (45) in the current study, more studies are needed with larger sample sizes before this can be used in routine clinical practice.

\section{References}

1. Saetung M, Kritsaneepaiboon S, Jarutach J. Main pulmonary artery-to-descending aorta ratio in computed tomography: cut-off value to diagnose pulmonary hypertension in children. Pol J Radiol 2021; 86: e87-e92.

2. Iyer A, Wells J, Vishin S, et al. CT scan-measured pulmonary artery to aorta ratio and echocardiography for detecting pulmonary hypertension in severe COPD. Chest 2014; 145: 824-832.

3. Karakus G, Kammerlander A, Aschauer S, et al. Pulmonary artery to aorta ratio for the detection of pulmonary hypertension: cardio- vascular magnetic resonance and invasive hemodynamics in heart failure with preserved ejection fraction. J Cardiovasc Magn Res 2015; 17: 79.

4. Madas S, Vittal D. Estimation of measured pulmonary artery to aorta ratio for prognosis of pulmonary hypertension in chronic obstructive pulmonary disease patients. Int J Adv Med 2016; 3 : 200-205.

Correspondence address:

Amarinder Singh, All India Institute of Medical Sciences, Sri Aurobindo Marg, Ansari Nagar, Ansari Nagar East, New Delhi, Delhi 110029, India,

e-mail:drasmalhi@gmail.com

Authors' contribution:

A Study design · B Data collection · C Statistical analysis · D Data interpretation · E Manuscript preparation · F Literature search · G Funds collection 